\title{
ORIGEN, TRAYECTORÍA Y EFECTIVIDAD DE LA FORMACIÓN DE ENSEÑANZA MEDIA TÉCNICO PROFESIONAL EN CHILE
}

DOI: http://dx.doi.org/10.1590/2236-3459/67121

\author{
Berta Servat Poblete \\ Pontificia Universidad Católica de Chile, Chile.
}

$\cos 8$

\begin{abstract}
Resumen
En Chile, los orígenes de la enseñanza técnica profesional se remontan al siglo dieciocho. Respondiendo a las emergentes necesidades de contar con recursos humanos especializados se establecieron los primeros establecimientos escolares de esta modalidad de enseñanza. A través del análisis de la trayectoria de la formación profesional se pueden apreciar diferentes momentos históricos, situaciones y características, referidas tanto a las instituciones y especialidades impartidas, como a su efectividad en cuanto a los requerimientos de la sociedad. El artículo da cuenta de parte del trabajo realizado en el Proyecto Fondecyt №1100473, institución a la que se agradece como también a quienes han colaborado en la realización del estudio.

Palabras clave: educación técnico profesional, trayectoria, efectividad, gestión directiva.
\end{abstract}

\section{ORIGEM, TRAJETÓRIA E EFETIVIDADE DA FORMAÇÃO DE ENSINO MÉDIO TÉCNICO PROFISSIONAL NO CHILE}

\begin{abstract}
Resumo
No Chile, as origens do ensino técnico profissional remontam ao século XVIII. Respondendo às necessidades emergentes de recursos humanos especializados, as primeiras escolas deste tipo de ensino foram estabelecidas. Através da análise da trajetória da formação profissional em diferentes momentos históricos, situações e características, envolvendo tanto as instituições e as especialidades, como sua eficácia em termos das exigências da sociedade. O artigo relata do trabalho realizado no Projeto Fondecyt №1100473, instituição que se agradece, bem como aqueles que têm colaborado no estudo.

Palavras-chave: educação profissional técnica, carreira, eficácia, gestão.
\end{abstract}

\section{ORIGIN AND EFFECTIVENESS OF CAREER TRAINING PROFESSIONAL TECHNICAL EDUCATION MEDIA IN CHILE}

\begin{abstract}
In Chile, the origins of vocational technical education dating back to the eighteenth century. Responding to the emerging needs of specialized human resources have the first schools of this type of education were established. Through the analysis of the trajectory of vocational training you can appreciate different historical moments, situations and characteristics, involving both institutions and given specialties, and its effectiveness in terms of the requirements of society. The article reports of the work done in the Fondecyt №1100473, project institution which is appreciated as well as those who have collaborated in the study. Keywords: professional technical education, career, effectiveness, management directive.
\end{abstract}




\section{ORIGINE, TRAJECTOIRE ET EFFICACITÉ DE LA FORMATION E PROFESSIONNELLE ENSEIGNEMENT TECHNIQUE MEDIA AU CHILI}

\section{Résumé}

Au Chili, les origines de l'enseignement technique professionnelle datant du XVIIle siècle. En réponse aux nouveaux besoins des ressources humaines spécialisées ont les premières écoles de ce type d'enseignement ont été établis. Vous pouvez Grâce à l'analyse de la trajectoire de la formation professionnelle apprécier les différents historiques des moments, des situations et des caractéristiques, impliquant à la fois des institutions et des spécialités données, et son efficacité en termes de besoins de la société. L'article rapports du travail effectué dans le Fondecyt $N^{\circ} 1100473$, Projet institution qui est appréciée aussi bien que ceux qui ont collaboré à l'étude.

Mots-clés: éducation technique professionnelle, carrière, efficacité, directive sur la gestion. 


\section{Introducción}

a educación técnico profesional (ETP) es una modalidad de aprendizaje escolarizado, presente en Chile desde antes de que se convirtiera en un país independiente del Reino de España. Durante el tiempo transcurrido ha pasado por distintos momentos e incorporado diversos cambios estructurales, los que, en alguna medida, han sido concomitantes a los procesos sociales, laborales y económicos existentes en el país en cada uno de sus momentos históricos.

La respuesta de este sector del sistema educacional a las necesidades de la sociedad en cuanto a contar con recursos humanos de nivel técnico y, por ende, a sus expectativas, han sido diferentes según la época no aportando siempre de similar forma ni efectividad. En ello, han influido una serie de factores, de distinta naturaleza, no sólo atingentes a las instituciones escolares, sino que en buena parte resultante de las posturas y acciones de las autoridades nacionales y sectoriales, como también de actores del mundo del trabajo y de la educación superior. Luego, se podría decir que en la trayectoria de la ETP han existido momentos más significativos que otros.

Sin duda, en la actualidad la necesidad de cambio cualitativo en este sector educacional es mayor que en tiempos pasados y, dada la conciencia que de ello se tiene, son exigidos desde diferentes sectores de la sociedad global, situación que en sus orígenes y durante gran parte de su trayectoria no existía.

En el país se están realizando esfuerzos relevantes para mejorar significativamente la efectividad de la modalidad de aprendizaje técnico profesional, lo que sin duda se encuentra en deuda. Dada esta realidad, es deseable la pronta incorporación de cambios estructurales y de contenidos, que no solo se enfoquen en los procesos internos de los establecimientos escolares, sino que también se extiendan hacia el medio ambiente externo. Ello, especialmente en lo pertinente a los contactos y asociaciones deseables de existir entre el establecimiento escolar y las organizaciones destino de sus egresados, tanto las de naturaleza laboral como educativa. Estos cambios no sólo son necesarios, sino que urgentes de materializarse al más breve plazo.

En este nuevo escenario, al Estado y sus organizaciones les cabe cumplir una función esencial, promoviendo y ejecutando los cambios que son necesarios, a la vez que asegurando su permanencia en el tiempo, de acuerdo a las características y exigencias propias de una sociedad moderna en la que en el mundo del trabajo, además de nuevos conocimientos imperan valores y pautas de comportamiento bastante diferentes a los existentes hace décadas y siglos atrás.

\section{Orígenes y trayectoria}

Es durante el siglo XVIII, cuando por primera vez en Chile se crea un establecimiento educacional de naturaleza técnico profesional. Efectivamente, en diciembre del año 1795 la Junta del Consulado de Chile, crea la Real Academia de San Luis, lo que se realizó por sugerencia de Manuel de Salas (1754-1841), destacado educador y diputado de la Nación. La misión de esta institución escolar fue formar especialistas de nivel técnico en las áreas de geometría, aritmética y dibujo, con el fin de aportar recursos humanos que potenciaran el desarrollo de la agricultura, el comercio y la minería. Esta iniciativa fue aprobada por el entonces Gobernador de Chile, Marqués de 
Avilés el 6 de marzo de 1797 (CALISTO; CUADRA; CUEVAS, 2014).

Es importante destacar que, en alguna medida, esta institución fue precursora del Instituto Nacional, establecimiento escolar científico humanista que teniendo sus orígenes en el siglo XIX, hasta nuestros días se ha mantenido como una institución pública emblemática de excelente nivel académico, lo que queda demostrado por destacados profesionales que han egresado de sus aulas, entre los que se cuentan varios Presidentes de la República, otras altas autoridades y destacadas personalidades nacionales.

Durante las primeros décadas de existencia de Chile como país independiente del Reino de España, una vez habiéndose superado los problemas políticos internos y de gestión propios de una naciente república y asegurada la institucionalidad establecida por la Constitución de 1833, las autoridades gubernamentales se ocuparon de dar al país un sistema educacional acorde a las necesidades de la población. Así es como se propició la fundación de una cantidad relevante de establecimientos de educación primaria, y conjuntamente con ello, surgió la necesidad de impartir enseñanza técnica a un segmento socio económico de la población que residía en las urbes, especialmente en la capital del país, la ciudad de Santiago. En el año 1844 siendo Presidente Manuel Bulnes Prieto (período 1841-1851), el entonces Ministro de Justicia Culto e Instrucción Pública, Manuel Montt Torres (1809-1880), planteó la urgente necesidad de crear en la capital, Santiago, un establecimiento escolar que impartiera enseñanza sobre oficios industriales en las áreas de carpintería, herrería, fundición y mecánica. Para concretar este proyecto, y dada la carencia de maestros en Chile que tuviesen las competencias requeridas se contrataron profesores traídos desde Francia. De esta forma se fundó la Escuela de Artes y Oficios bajo la dirección del ciudadano francés Jules Jariez -ingeniero y pedagogo- el 8 de agosto de 1849 (BIBLIOTECA NACIONAL DE CHILE, 2016).

Los primeros estudiantes debían tener entre 15 y 18 años de edad, saber leer y escribir y conocer las operaciones aritméticas. Sin embargo, aparte de una intensa formación práctica en talleres, los jóvenes también estudiaban álgebra, geometría descriptiva, trigonometría, dibujo técnico, mecánica industrial, física y química, aparte de castellano, historia y geografía y otros cursos básicos. Esta formación, que originalmente duraba cuatro años, aumentó a cinco en 1858. Los egresados recibían la calificación de 'aprendiz' (BIBLIOTECA NACIONAL DE CHILE, 2016).

La alta calidad de la enseñanza que se impartía en esta institución, generó que se expandiera fuera de la capital, Santiago, como también que contara con alumnos provenientes de otros países de latino américa. Este establecimiento educacional gozó de un gran prestigio, cumpliendo una importante función en un momento de la vida de la República en que la educación formal no era masiva, ni tampoco existía suficiente conciencia de la importancia de ello. En el año 1886, como consecuencia tanto del prestigio adquirido, como del relevante aumento de su alumnado, se inició la construcción de un nuevo edificio el cual fue inaugurado en el año 1893 (BIBLIOTECA NACIONAL DE CHILE, 2016).

Es importante señalar que el proceso de expansión de la Escuela de Artes y Oficios de Santiago tuvo su correlato en otras instituciones similares que se fundaron en provincia. Efectivamente, a la época existían las Escuelas de Minas en las ciudades nortinas de Antofagasta, de Copiapó y de La Serena, y las Escuelas Industriales en 
ciudades del sur del país, Concepción, Temuco y Valdivia. Las especialidades de formación técnica a los alumnos correspondían a los particulares requerimientos de recursos humanos de nivel técnico de las regiones en que se encontraban, aportándose así desde el sistema escolar al desarrollo económico de las provincias en particular y del país en su conjunto (BIBLIOTECA NACIONAL DE CHILE, 2016).

A partir del año 1912 la Escuela de Artes y Oficios formó profesionales titulados como sub ingenieros industriales, que luego fueron conocidos como técnicos. En esos tiempos la institución otorgaba dos niveles de formación: Uno, correspondiente a nivel secundario que otorgaba el Grado de Oficios y el otro nivel, de nivel terciario que otorgaba el Grado de Técnicos. Después de realizarse un cambio en los planes y programas de estudio, lo que sucedió en la década de 1960, los técnicos de esta escuela, que desde el año 1947 se había incorporado a la Universidad Técnica del Estado, pasaron a llamarse Ingenieros de Ejecución (MUÑOZ, 1987).

A continuación, y con el propósito de destacar el nivel de formalización de la institución y las particularidades existentes, de acuerdo a la época histórica, se transfiere parte del Reglamento para la Escuela de Artes y Oficios, dictado por el Supremo Gobierno, el 22 de Enero de 1864, el cual estaba integrado por 15 Títulos, y una gran cantidad de artículos en cada uno de ellos. El documento resulta interesante de revisar para situar y apreciar desde una mirada histórica, la naturaleza de la institución, sus características y procedimientos.

Los títulos del Reglamento son los siguientes: Título I. Objeto y Personal de la Escuela; Título II. Empleados. Del Director; Título III. Del Inspector Jefe; Título IV. De los Inspectores; Título V. De los Profesores; Título VI. Consejo de Profesores; Título VII .Del Tesorero; Título VIII. Del Ingeniero de los Trabajos; Título IX. Del Guarda Almacenes; Título X. De los Maestros de Taller; Título XI. Del Maquinista; Título XII. Del Capellán; Título XIII. Del Médico; Título XIV. Del Mayordomo; Título XV. De los Alumnos; Titulo XVI. De la Distribución del Tiempo; Título XVII. De la Enseñanza; Título XVIII. De los Exámenes; Título XIX. De los Premios; Título XX. Delitos y Penas; Título XXI. Objetos que Fabrica la Escuela; Título XXII. Disposiciones Transitorias (SUPREMO GOBIERNO DE CHILE, 1864).

Especial mención se hará del Título XV. "De los Alumnos, que teniendo 13 artículos, especifica lo siguiente:"

Art. 38. Las plazas de alumnos que anualmente quedaren vacantes se llenarán con jóvenes tomados de las diferentes provincias de la República, a discreción del Gobierno.

Art. 39. Para ser alumno de la Escuela se requiere: 1․ Tener de diez i seis a veintiún años de edad, justificada con la fe de bautismo o con información de testigos. 2ำ Acreditar buena conducta. 3o Tener buena constitución física, justificada con certificado de médico; $4^{\circ}$. Saber correctamente leer i escribir i las cuatro primeras operaciones de la aritmética, el sistema métrico de pesos i medidas i nociones elementales de catecismo, de religión i de gramática castellana; $5^{\circ}$ No tener defectos físicos que sean incompatibles con la profesión a que se va a dedicar.

Art. 40. La elección de alumnos se hará en concursos que tendrán lugar en las cabeceras de cada departamento, con tres meses de anticipación a la época fijada, para la apertura del nuevo año escolar.

Art. 41. Los concursos de que se habla en el artículo anterior tendrán lugar ante comisiones compuestas de tres personas idóneas nombradas por el gobernador. 
El visitador de escuela formara parte de la Comisión del departamento donde estuviere.

Art. 42. Los solicitantes presentaran a la comisión los documentos a que se refieren a los incisos $1^{\circ}, 2^{\circ}$ i $3^{\circ}$ del art. 39 , para que ella los califique.

Art. 43. Del resultado de los exámenes se levantará, una acta, en la cual se expresará el grado de aprovechamiento de cada uno de los examinados i las observaciones particulares que la junta examinadora juzgue necesario hacer. La acta, acompañada de lo justificativos antedichos, se pasará al Gobierno por conducto del Intendente respectivo.

Art. 44. Decretada la incorporación de los alumnos, puede el director del establecimiento, durante el primer trimestre, que se considerará de prueba, pedir al Gobierno la separación de aquellos que manifiesten mal carácter, desaplicación o salud delicada.

Art. 45. Los jóvenes que hayan de incorporarse como alumnos de la Escuela de Artes se comprometerán a trabajar en su respectiva profesión después de concluido su aprendizaje i durante el término de seis años en el lugar que el Gobierno les fije. Si fueren separados de la Escuela por mala conducta o desaplicación calificadas por el Director, devolverán al fisco la cantidad que se hubiere gastado en su educación como también los gastos de viaje y otros que se hubieren hecho a favor de ellos; según cuenta que pasará el Director de la que podrá reclamarse ante el Gobierno.

Art. 46. Los padres o curadores de los alumnos asegurarán el cumplimiento de la obligación que habla el artículo anterior con una fianza mancomunada y solidaria calificada por el respectivo Gobernador.

Art. 47. La fianza se otorgará por escritura pública, i se mandará al Ministerio de Instrucción Pública una copia autorizada de ella junto con los documentos mencionados en el artículo 43.

Art. 48. Tres días después de haber terminado los exámenes públicos, que anualmente deben rendirse en la Escuela, el Director dará cuenta al Ministerio de Educación Pública, de los alumnos que hubiesen concluido sus estudios, a fin de que el Gobierno disponga de ellos.

Art. 49. Los alumnos a tiempo de entrar a la Escuela i siempre que circunstancias especiales, a juicio del Director, no se opongan a ello, podrán elegir los talleres que más les agradare de entre los que a continuación se espresan: herrería, fundición, calderería i cualquiera de los de carpintería. El Director al fin del primer año decidirá cuales de los herreros pueden pasar al taller de mecánica i distribuirá a los aprendices de carpintería entre los diversos talleres de esa profesión según sus aptitudes. Escuela de Artes y Oficios. Santiago de Chile, 1864.

(SUPREMO GOBIERNO DE CHILE, 1864, p. 21-23)

Ahora bien, en la segunda parte del siglo XIX, en el mes de Enero de 1887, durante el gobierno del Presidente Don Manuel Balmaceda, se crea la Escuela de Artes y Oficios para mujeres, impartiéndose contenidos en las siguientes áreas: comercial, moda, lencería y costura corriente, bordado, guantería, cartonaje y marroquinería, cocinería, lavado y planchado, y dibujo (SUPREMO GOBIERNO DE CHILE, 1888).

A continuación se transcribe parte de lo establecido textualmente, al respecto.

\section{ESCUELA DE ARTES I OFICIOS PARA MUJERES.}

El Supremo Gobierno sometió a un estudio del Consejo de Enseñanza Agrícola e Industrial el proyecto sobre creación de una escuela de artes i otra de oficios para mujeres, presentado por la Sociedad de Fomento Fabril en Agosto del año anterior. El Consejo de Enseñanza aceptó ese proyecto con el entusiasmo que naturalmente debía despertar una idea cuya realización prestará considerables beneficios a la mujer, que hasta ahora carecía de los medios necesarios para adquirir sin sacrificios conocimientos industriales que le permitieran asegurarse un trabajo remunerativo i propio de su naturaleza. Por otra parte, la industria perdía un poderoso elemento con la falta de aplicación de la mujer a ciertos trabajos que le son peculiares i en los que se perfecciona fácilmente. Esa misma falta de aplicación nos hace pagar hoy un subido tributo a la internación extranjera por artículos que representan un trabajo de mujer que puede i debe ser ejecutado en 
el país. Dada esta situación, el Consejo de Enseñanza no podía menos de prestar su apoyo al proyecto que se le consultaba. El deseo de la Sociedad era que se fundasen dos escuelas para mujeres: una industrial con once secciones, i otra de oficios con tres. El gasto anual que hubiera demandado ponerlo en práctica se calculaba en 34,400 pesos. Como no pudiere disponerse en el presupuesto de este año sino de 25,000 pesos hubo necesidad de suprimir aquellas secciones que se consideraron menos necesarias i de hacer una sola de las dos escuelas proyectadas. (SUPREMO GOBIERNO DE CHILE, 1888, p. 43)

A pesar de la existencia de estas y otras instituciones, en Chile aún se estaba lejos de que la enseñanza técnica respondiera a la existencia de políticas de estado, ya que su origen obedecía principalmente a iniciativas de instituciones y o personas que, sin duda, en alguna medida fueron actores visionarios. Así es como, hubo que transcurrir un tiempo, para que se institucionalizara desde el Estado el propósito de crear centros de formación técnica.

Para ello nos debemos remontar a los inicios del siglo $\mathrm{XX}$, cuando durante la Presidencia de Don Pedro Montt (período 1906-1910), en el año 1908, se realizó un ordenamiento de la enseñanza secundaria, estableciéndose dos niveles. Por una parte, las instituciones que impartían formación general $y$, por otra parte, aquellas que fueron concebidas como escuelas comerciales, técnicas o de oficio (LÓPEZ, sin fecha citado en Calisto et al., 2014).

Más adelante y a partir de reformas que se fueron realizando en la educación formal, entre los años 1935 y 1950, y como una estrategia para impulsar la industrialización del país, desde el Estado se efectúan acciones tendientes a optimizar, tanto cuantitativa como cualitativamente la enseñanza técnica de nivel secundario, ello como respuesta a la creciente necesidad de industrialización de la sociedad chilena. Así es como, mediante estas reformas educacionales, se le dio un importante impulso a la enseñanza de carácter técnico (LÓPEZ, sin fecha citado en Calisto et al., 2014).

Efectivamente, luego de la depresión económica que se produjo en Chile, en los años 30 del siglo $\mathrm{XX}$, generada entre otros factores por el descubrimiento del salitre sintético que produjo una significativa disminución de la productividad de la explotación del salitre, mineral que sustentaba la economía nacional, el Estado comenzó a intervenir directamente en el desarrollo de la industria nacional, lo que favoreció el mejoramiento de las instituciones escolares de nivel técnico, las cuales a la fecha y como sigue ocurriendo hasta nuestros días han sido, en su mayoría, financiadas por el Estado y administradas y/o supervisadas por el Ministerio de Educación.

Durante la década del cuarenta los estudiantes de las escuelas técnicas agrupados en la Federación de Estudiantes Mineros e Industriales de Chile, realizaron diversas actividades para transformar estos establecimientos de enseñanza secundaria en instituciones universitarias. Dichas aspiraciones se materializaron el 19 de Abril de 1947, durante el gobierno del Presidente Gabriel González Videla (período 1946-1952), quien fundó mediante el Decreto № 1831, la Universidad Técnica del Estado sobre la base de los centros enseñanza de la Escuela de Artes y Oficios, en Santiago y con sedes en regiones (BIBLIOTECA NACIONAL DE CHILE, 2016).

Este hecho representa un hito histórico relevante en la trayectoria de la formación técnica en Chile, dado el significativo aporte de esta institución de educación superior. Ello, tanto para el desarrollo del país al formar recursos humanos en áreas de importancia 
estratégica, como también por facilitar la movilidad social de sectores más vulnerables socioeconómicamente que el alumnado de las universidades tradicionales.

La participación en calidad de profesionales de los egresados de la Universidad Técnica del Estado ha sido muy relevante, por haberse constituido esta casa de estudios en una de las universidades más prestigiosas del país.

Prueba de ello es la participación que les ha cabido a estos profesionales, entre los años 1952 y 1964, en la elaboración y concreción de proyectos eléctricos, petroleros, siderúrgicos, químicos y pesqueros, como también en la mayoría de los demás proyectos industriales llevados a cabo por la Corfo - Corporación de Fomento- creada durante el segundo gobierno de Don Arturo Alessandri Palma (período 1932-1939), momento en que se inició el intenso debate del devenir económico e industrial del país, que en lo económico era de subdesarrollo y en lo social existencia de profunda desigualdad entre la población, debido a que la mayoría de las personas no satisfacían sus necesidades básicas, incluidas las instruccionales (MINEDUC, 2013).

En el año 1934 volvió a establecerse un organismo que se había creado en 1931 con el nombre de Consejo de Economía Nacional. Así es como el 29 de abril de 1939, se promulga la "Ley N 6.434 de Reconstrucción, Auxilio y Fomento de la Producción" que crea la Corporación de Fomento de la Producción y la Corporación de Reconstrucción y Auxilio.

Como una primera y relevante misión fue encomendado a Corfo el reclutamiento de profesionales expertos, especialmente de profesión ingeniero y economista. Entre otros notables especialistas se encuentran Guillermo del Pedregal, Raúl Zorrilla, Oscar Gajardo, Eduardo Figueroa Geisse, Mario Sarquis Yazigi, Guillermo Moore Montero, Germán Pico Cañas. La misión inicial encomendada por la autoridad fue la de realizar una labor de evaluación y catastro de los recursos naturales de Chile y posteriormente planificar la explotación más adecuada de los mismos. De este trabajo surgieron los siguientes planes: de Fomento de la Producción, de Producción de la Energía Eléctrica, de Acción Inmediata para la Agricultura y Explotaciones afines, de Fomento Industrial y de Acción Inmediata de Comercio y Transporte. Dichas estrategias de gestión fueron fundamentales para el fomento de la industrialización del país, marcando la primera década de Corfo. Es importante señalar la relevancia del incentivo que se realizó a las exportaciones, especialmente de la producción agrícola (BIBLIOTECA NACIONAL DE CHILE, 2016).

En lo que respecta a la misión de la Universidad Técnica del Estado, en cuanto a la preparación de profesionales de alto nivel, orientados preferentemente al servicio de sectores de la economía que no cubrían los profesionales egresados de las universidades tradicionales, en gran medida ello llegó a su fin durante el Gobierno Militar.

Efectivamente, en la década del 70 se realizó una separación de las sedes de la universidad, afectando a la que funcionaba en Santiago y a las de regiones, otorgándoles una misión diferente a la que hasta el momento tenía la universidad, asemejándolas al resto de las integrantes del Sistema de Educación Superior. Ello implicó que la universidad dejara de ser una entidad de naturaleza técnica o tecnológica. Se denominó Universidad de Santiago a la sede que operaba en la capital. Lo mismo ocurrió con las ex sedes que funcionaban en provincias o regiones, las cuales a partir de ese momento se constituyeron en instituciones individuales. 
Ahora bien, durante la década del 60 , y luego de una serie de modificaciones realizadas a la formación técnica, se establece en Chile un nivel primario de 6 años de escolaridad y una educación secundaria de otros 6 años para los establecimientos científico-humanistas. Respecto a los establecimientos que impartían formación técnica, se estableció la educación secundaria técnica con una duración de 7 años para los institutos comerciales y las escuelas agrícolas y 5 años para las escuelas industriales, las escuelas técnicas femeninas y las escuelas normales (MINEDUC, sin fecha citado en Calisto et al., 2014).

Tanto los liceos como las escuelas agrícolas, industriales, técnicas femeninas y los institutos comerciales tenían como propósito que los alumnos se incorporaran al mundo del trabajo una vez egresados o continuaran estudios en la educación superior. Dicha dualidad, hasta el presente no se ha logrado como es debido.

Las escuelas normales eran las instituciones formadores de maestros para la enseñanza básica o primaria, por lo que su destino inmediato siempre fue el laboral, desempeñando, sus titulados, funciones como educadores en el nivel de primaria o básica en los colegios del país.

Durante el año 1965, en el Gobierno de Don Eduardo Frei Montalva (período 1964-1970), se realizó una nueva reforma. La educación primaria aumentó de 6 a 8 años de estudios, denominándose a este nivel Educación General Básica. Con ello, se pretendía aumentar la escolaridad de los sectores más vulnerables. Para la educación secundaria se fijaron 4 años de escolaridad. En este nivel de estudio, se estableció la diferencia entre la enseñanza científico humanista y técnico profesional, siendo el propósito de esta última modalidad la capacitación de los alumnos para su futuro desempeño laboral en los distintos oficios y funciones técnicas de nivel medio, para así dar satisfacción a exigencias del desarrollo económico, social y cultural del país. También se estableció el propósito de preparar a los alumnos para que continuaran estudios en la Educación Superior. Hasta el momento presente, esta última vía ha sido muy poco fecunda, en términos del acceso de los egresados. Ello, especialmente dadas las especiales características socio económicas del alumnado de los establecimientos técnicos, como también la insatisfactoria preparación obtenida en éstos, en los contenidos cognitivos requeridos para continuar estudios superiores, la cual es significativamente menor a la de quienes han cursado la enseñanza media científico humanista (MINEDUC, sin fecha citado en Calisto et al., 2014).

Concomitante con estos cambios estructurales, los establecimientos que impartían enseñanza técnico profesional pasaron a denominarse Liceos, cambiando su nombre original de Escuela, lo que se institucionalizó mediante el Decreto № 300, del año 1981. En dicha norma legal se establecieron dos momentos o ciclos de enseñanza media, uno integrado por el primero y el segundo año medio, comunes tanto para la enseñanza científico humanista como para la enseñanza técnico profesional y el segundo ciclo integrado por el tercero y cuarto medio. Al inicio de éste, los alumnos optan según sus intereses. Se estableció un plan común para ambas modalidades de enseñanza y planes diferenciados de especialidad para la enseñanza técnico profesional (CALISTO et al., 2014).

Con estas modificaciones se restaron años y horas a la enseñanza técnica, lo que podría constituir una de las causas del actual nivel deficitario de este tipo de formación. 
Durante la década de los 80 se produjo un cambio que resultó ser de corta duración en la modalidad técnico profesional, ya que se fue implementando un quinto año de enseñanza media en la mayoría de los liceos polivalentes y técnicos profesionales propiamente tales, con el propósito de reforzar la formación técnica. Sin embargo en el año 1987, el Ministerio de Hacienda eliminó el financiamiento que se había otorgado con dicho propósito, volviéndose a los 4 años de enseñanza media. Es importante señalar que la formación específicamente de carácter técnico solo se imparte en los 2 últimos años de este nivel. También en esos años se amplió la libertad y atribuciones otorgadas a los liceos técnicos profesionales en cuanto a la elaboración de propuestas de nuevas especialidades, de acuerdo a los resultados de estudios de mercado realizados en sus comunidades. Sin embargo, un estudio de la Universidad de Santiago demostró que ello no fue así (COLEGIO DE PROFESORES DE CHILE, 2007, citado en Calisto et al., 2014).

Ello, no se realizó en esos momentos y tampoco constituye una práctica en nuestros días. Lo cual representa una de las causas probables de la falta de efectividad de la formación de EMTP. Efectivamente, evaluaciones orientadas a cuantificar oportunamente las necesidades cuantitativas y cualitativas de técnicos requeridas en las ciudades, regiones y en el conjunto del país, constituye aún un tema pendiente.

Entre los años 1981-1987 la educación media experimentó un crecimiento cuantitativo importante, en lo pertinente a la cantidad de alumnos que se incorporaron a los establecimientos. En este escenario, fue aún más significativo el aumento en la enseñanza técnico profesional, ya que cuatro de cada cinco nuevos alumnos prefirieron esta modalidad de enseñanza.

Continuando con esta tendencia, la participación relativa de estos alumnos en la matrícula total de la enseñanza media aumentó a 44\% en el año 1997 (MINEDUC, 1998). En la actualidad la situación no ha variado significativamente.

Una vez reestablecido el régimen de gobierno democrático, en el año 1990 el Ministerio de Educación realizó una evaluación de la situación de la educación media técnico profesional, y con el propósito de mejorar la efectividad de esta modalidad en términos de satisfacción de las necesidades del país en general y de sus usuarios en particular, se estableció lo que a continuación se señala.

\begin{abstract}
Mejorar la calidad de la educación técnico-profesional, a través de acciones de perfeccionamiento y actualización de los profesores y de implementación del equipamiento de los talleres y laboratorios, para proporcionar una formación técnica acorde a las necesidades del sistema productivo, lo cual implica entre otros aspectos, el modernizar la educación para el trabajo mediante la introducción de modalidades, métodos y procedimientos que puedan atender las demandas del sistema productivo y la rapidez del cambio tecnológico, para hacer concordante la formación de recursos humanos calificados y su incorporación al campo laboral. (MINEDUC, 1992, p. 3)
\end{abstract}

Para llevar a efecto este gran objetivo, se formularon los siguientes proyectos:

1. "Programa de emergencia para la modernización de la Educación Técnico Profesional que se imparte en establecimientos municipalizados (D.F.L.1/3063), que es un convenio entre Chile y España..."

2. "Modernización Tecnológica y Curricular en la Educación Técnico Profesional Municipalizada". "Convenio Chile-Italia." 
3. "Aplicación de un plan de desarrollo de establecimientos de educación técnicoprofesional, articulado con sectores productivos y de servicios". "Convenio Chile-Francia."

4. "Modernización y desarrollo de la Educación Técnico Profesional que se imparte en Liceos Comerciales Municipalizados". "Convenio Chile-Francia."

5. "Aplicación experimental del sistema dual en establecimientos de Educación Media Técnico- Profesional.". "Convenio Chile-Alemania."

6. "Misión Británica de expertos en Educación Técnica". "Convenio Chile-Gran Bretaña."

(MINEDUC, 1992, p. 4-6)

Durante la década de 1990, el Ministerio de Educación realizó una evaluación de las especialidades que se otorgaban a la fecha (403), estableciéndose una radical disminución del número de éstas, reduciéndose en un primer momento a 63 y posteriormente a 44. Se aumentaron 2 especialidades en el año 2001, quedando en 46 (CALISTO et al., 2014).

Conjuntamente con este cambio de estructura, se introdujo una importante modificación cualitativa en el sistema de enseñanza técnica. Esta consistió en un cambio de paradigma en términos de los propósitos de este tipo de enseñanza, cambiando el de capacitar para la ejecución de un trabajo o tarea específica, a una enseñanza que le posibilitara al joven una vez incorporado al mundo del trabajo, adaptarse rápidamente a los cambios tecnológicos que se suceden en dicho espacio laboral (CALISTO et al., 2014).

De acuerdo a este nuevo propósito, surge en Chile la implementación del enfoque pedagógico basado en competencias, tanto competencias básicas como específicas, lo cual cambia la modalidad asignaturista por una estructura modular para desarrollarse en cada una de las especialidades. Desde el Mineduc se proporciona a los establecimientos educacionales técnicos una propuesta de aprendizajes esperados y criterios de evaluación (CALISTO et al., 2014).

Dicho enfoque representa un hito importante en la trayectoria de la enseñanza técnico profesional, permaneciendo hasta nuestros días.

El modelo curricular basado en competencias representa la ideología curricular de eficiencia social de Schiro, considerándose que la educación tiene como propósito el capacitar a los estudiantes para que así estos puedan contribuir a la sociedad de manera eficiente. Su meta principal es entrenar a los estudiantes en habilidades y procedimientos que estos necesitarán en sus futuros trabajos de modo de desempeñarse de manera productiva, perpetuando el funcionamiento de la sociedad. En esta ideología se postula que la esencia de los estudiantes reside en sus competencias y en las actividades que son capaces de realizar (SCHIRO, 2008).

Los cambios de carácter curricular y metodológicos establecidos en la enseñanza media técnico profesional, se realizaron desde el Estado una vez iniciado el primer gobierno elegido por los ciudadanos, desde la interrupción de la democracia en el año 1973. Estos, se originaron en la evaluación realizada acerca de la efectividad de la formación profesional de nivel técnico proporcionada por las instituciones de educación secundaria en el país. Para subsanar los déficits instruccionales y formativos detectados, de esta modalidad educativa, se elaboraron los cambios pertinentes, de forma y contenido. Ello, para responder desde este sector de la educación a las crecientes necesidades de recursos humanos requeridos por el sector laboral, como también a las 
competencias exigidas por el sistema superior de educación. Tanto unas como otras no coincidían con las competencias laborales y cognitivas de los egresados de los establecimientos escolares que impartían formación técnica.

Principalmente se trabajó en la incorporación de modificaciones relevantes de tipo curricular, como también en la reorganización del sistema de especialidades en las que se forman los alumnos.

En este escenario, ha resultado muy importante el cambio de paradigma incorporado a la formación técnica, principalmente consistente en la preparación de los jóvenes en competencias que les permitan desempeñarse con éxito en el sector laboral, en un escenario nacional y mundial caracterizado por los avances en el plano del conocimiento científico y tecnológico, y por la globalización cada vez mayor de la economía. Se optó por el reforzamiento significativo del contenido de los planes de estudio, como también de las especialidades que se imparten.

El propósito principal ha sido reforzar las oportunidades de los alumnos egresados, en cuanto a la obtención de un trabajo adecuadamente remunerado y en el que puedan hacer carrera, como también, si responde a sus intereses, poder continuar estudios en el sistema de educación superior, especialmente en centros de formación técnica e institutos profesionales.

En este escenario, se presenta como una alternativa metodológica potente de formación técnica la modalidad Dual, la que generada en Alemania se ha adaptado, con éxito, a la realidad chilena en establecimientos técnicos profesionales. Sin embargo, en Chile estos no alcanzan a cubrir el $10 \%$ del total de centros TP por lo que hasta el momento no se ha extendido como debiese esta manera de operar metodológicamente en la enseñanza técnica.

En documento elaborado por el Ministerio de Educación se establece respecto a la formación Dual, lo siguiente:

[...] es un modelo de alternancia curricular centrada en la articulación entre la educación y el sector productivo que se desarrolla en dos lugares de aprendizaje complementarios: el establecimiento educacional y la empresa. Los alumnos pueden asistir en regímenes de tres días al liceo y dos días en la empresa, o bien de una semana en el liceo y una semana en la empresa. Las empresas reciben a uno o más "aprendices" durante dos años seguidos, a partir de $3^{\circ}$ Medio respetando su condición de alumnos del liceo. El modelo se implementó en Chile en 1992, como una iniciativa de la Sociedad Alemana de Cooperación Técnica (GTZ) y el Gobierno de Chile para fortalecer la EMTP en términos de calidad y vinculación con el mundo productivo. (MINEDUC, 2011, p. 19)

El aporte de la modalidad Dual, principalmente radica en que durante el proceso de formación profesional de los alumnos, estos se vinculan real y significativamente con el mundo del trabajo, destino inmediato o mediato de los egresados. Ello, a partir del contacto que se establece entre los jóvenes con las empresas durante todo el proceso educativo, realizando práctica profesional. Esta, no es terminal o al final del período de formación. Es supervisada por empleados de la empresa en la que la realizan, con la consecuente adquisición por parte de los alumnos de metodologías y procedimientos técnicos de punta, como también, en lo que respecta a la formación tan exigida por los empleadores, las llamadas competencias blandas. Estas se refieren a la socialización en valores y pautas de comportamientos exigibles en el mundo del trabajo en el escenario 
planetario actual.

De esta manera, la formación profesional adquiere la pertinencia de contenidos y procedimientos requerida por los empleadores respecto a la preparación de los futuros trabajadores, tanto en los planos del ser como del conocer y del hacer.

En lo que respecta a la incorporación de contenidos curriculares orientados a la enseñanza profesional de "calidad", es importante señalar que desde hace años el Ministerio de Educación ha tenido dicho propósito, materializado más o materializado menos. Por ello, en Chile como en otros países se ha procurado contar con sistemas de aseguramiento de la calidad. Respecto a éstos, se transcribe lo siguiente:

[...] sistemas de aseguramiento de calidad que permitan monitorear el cumplimiento consistente de ciertos requisitos considerados necesarios para la efectividad de los procesos formativos. Estos requisitos se definen en términos de estándares y procedimientos a ser monitoreados, los que en el caso de no ser cumplidos deben ser analizados y solucionados. Al respecto se distingue entre estándares de entrada, proceso y salida. Los primeros dan cuanta de los recursos que deben estar disponibles en una organización formativa (equipo de personas, materiales, infraestructura), mientras que los de proceso se refieren a las actividades que se desarrollan dentro de la organización. Por su parte, los estándares de salida dan cuenta de los resultados (tasas de titulación, logros de aprendizajes) del conjunto de procesos y por lo tanto establecen el nivel de desempeño a ser alcanzado. (MINEDUC, 2011, p. 22-23)

Se intentó materializar este sistema de aseguramiento de la calidad, mediante el Programa Chile Califica, a partir de la acreditación que se realizó de las especialidades técnicos profesionales, enfocándose en estándares de entrada. Entre los años 2003 a 2009 , voluntariamente el $76 \%$ de las especialidades ofertadas pasaron por este proceso. De éstas fueron acreditadas el $70 \%$. Sin embargo, sus efectos en cuanto a mejoras de la calidad fueron limitados, debido a que no se cumplió con el ciclo completo de evaluación. En el año 2009, se cerró el Programa Chile Califica, discontinuándose la línea de Acreditación de Especialidades TP (MINEDUC, 2011).

Retomando el análisis curricular, hay que señalar que en el año 1998 se realizó una nueva reforma (Decreto 220), mediante la cual se organiza la Enseñanza Media Técnico Profesional (EMTP), en 14 sectores económicos y 46 vías de especialización, estableciéndose planes diferenciados en el currículum, a partir de $3^{\circ}$ medio. Por tanto, esta modalidad de enseñanza comparte un currículum común con la Enseñanza Científico Humanista en los niveles de $1^{\circ}$ y $2^{\circ}$ medio (MINEDUC, 2011).

De acuerdo al decreto 220, el marco curricular de la formación diferenciada técnica profesional se expresa en términos de objetivos terminales fundamentales, agrupados en Perfiles de Egreso, correspondientes a las 46 vías de especialización. Estos perfiles representan una base común de formación, y expresan lo mínimo y fundamental que debe aprender todo estudiante que curse una especialidad. Para su implementación, el Ministerio de Educación puso a disposición de los establecimientos escolares programas de estudio en estructura curricular modular, con enfoque en competencias laborales que contienen una propuesta de aprendizajes esperados y criterios de evaluación. El desarrollo de estos módulos no es obligatorio para los liceos, otorgándoles la libertad de generar sus propios programas para las especialidades que imparten, pero si deben dar cuenta de los Perfiles de Egreso asociados a las 
especialidades (MINEDUC, 2011).

En el año 2006, se realizaron nuevos ajustes al marco curricular, iniciándose con estudios de pertinencia para 21 especialidades. A partir de ello, se introdujeron cambios en los Perfiles de Egreso, y posteriormente, en los Planes y Programas de Estudio, orientados a fortalecer la formación de los alumnos en "competencias" (MINEDUC, 2011).

El Ministerio de Educación, en el año 2013, elaboró nuevas Bases Curriculares (perfiles de egreso) para la formación diferenciada Técnico Profesional. Dicha modificación de los perfiles acoge las nuevas exigencias curriculares que establece la Ley General de Educación (LGE) y busca, además, adecuar el currículum a las demandas de los sectores productivos del país para mejorar así la empleabilidad y condiciones laborales de los alumnos y brindarles mejores oportunidades para la continuidad de estudios (MINEDUC, 2013).

De acuerdo a lo anterior, se crearon dos especialidades nuevas, se fusionaron algunas de las especialidades anteriores y se elaboró un nuevo ordenamiento de ellas, creando nuevas menciones con lo cual en la actualidad estas Bases Curriculares definen 34 especialidades agrupadas en 15 sectores económicos. Algunas de las especialidades ofrecen dos o tres menciones. Esta organización curricular implica un tronco común de competencias a ser desarrolladas durante el primer año de especialidad, y otras competencias diferenciadas para cada mención, a ser desarrolladas en el segundo año, sumando en total 17 menciones. Los sectores son los siguientes: Maderero; Agropecuario; Alimentación; Construcción; Metalmecánica; Electricidad; Marítimo; Minero; Gráfico; Confección; Administración; Salud y Educación; Química e Industria; Tecnología y Comunicaciones; Hotelería y Turismo (MINEDUC, 2013).

Se descontinuaron 9 especialidades (Secretariado, Ventas, Procesamiento de la madera, Mecánica de aeronaves, Atención social y recreativa, Celulosa, Tejidos, Textil, y Productos del cuero), Para cada especialidad discontinuada se ofreció la alternativa más demandada por el mercado. Se agregaron dos especialidades nuevas (Programación y Conectividad y Redes), integrando el nuevo sector Tecnología de las comunicaciones.

Los perfiles de egreso están precedidos de una breve descripción del contexto laboral de cada especialidad. Este contexto describe la actividad económica asociada a la especialidad, principalmente los indicadores que dan cuenta de su dinamismo y potencial de crecimiento, los desafíos que enfrenta, el tipo de empresas que conforman el campo laboral para los egresados y los productos que se esperan de su trabajo. (MINEDUC, 2013, p. 11)

Para cada especialidad se ha definido un conjunto de objetivos de aprendizaje a ser logrados al final de los dos años de formación diferenciada. Estos objetivos configuran un perfil de egreso, que expresa lo mínimo y fundamental que debe aprender todo alumno o alumna del país que curse una especialidad. No se trata de un perfil ocupacional, tal como se lo entiende en los procesos de capacitación (centrado en las tareas que se debe cumplir en un puesto de trabajo), sino de un delineamiento de competencias que preparan para iniciar una vida de trabajo y que se espera domine un estudiante al egresar de la EMTP. (MINEDUC, 2013, p. 12)

Los perfiles de egreso contemplan dos categorías de objetivos de aprendizaje: unos aluden a las competencias técnicas propias de la especialidad o de la mención, en tanto otros se refieren a las competencias que son de carácter general para el mundo laboral. Estas son comunes a todas las especialidades, ya que son requeridas en los desempeños de todos los técnicos, - y de todos los 
trabajadores - independientemente del sector productivo al que esté vinculada la especialidad. Algunas de estas competencias trascienden al mundo laboral, y son aplicables a otros ámbitos de la vida de las personas, pues apuntan a su formación integral. (MINEDUC, 2013, p. 12)

Las nuevas bases curriculares fueron aprobadas en Julio del año 2013 por el Consejo Nacional de Educación. Estas empezaron a regir de manera flexible en el año 2014 y son obligatorias a partir del año 2015.

Actualmente, y en el marco de la reforma a la educación que se está realizando en el país, y para fortalecer la educación técnica, el Ministerio de Educación creará una instancia público-privada para saber cuántos trabajadores se necesitan por región y que calidad deben tener éstos. A la vez, se definirán estrategias a desarrollar con el fin de lograr los propósitos establecidos. De hecho, el 21 de Mayo de 2016 la Presidenta de la República anunció la creación de un Consejo Nacional de Formación Técnico Profesional, con el objetivo de fortalecer a este sector educativo para mejorar la productividad, competitividad e innovación del país. Esta instancia pretende institucionalizar la colaboración pública privada, en la que los sectores productivos podrán identificar el tipo de técnicos que se necesitan, de tal forma que, luego, los liceos industriales y los centros técnicos los formen.

El Consejo desarrollará una política educacional, contando con la participación de los Ministerios del Trabajo y de Economía y de instituciones tales como Corfo, Sence y Chile Valora, los que proporcionaran una "mirada país" acerca de los sectores estratégicos que son necesarios de desarrollarse. También se incorporaran los gremios de la industria, como la Confederación de la Producción y del Comercio (CPC), y la Sociedad de Fomento Fabril (Sofofa), organizaciones que aportaran mediante la cuantificación de los requerimientos de trabajadores por región, de que tipo deben ser y que conocimientos deben tener. La política que se elaborará tendrá tres pilares: asegurar la calidad de la educación, de tal modo que sea altamente competitiva, flexible, innovadora y estimule el emprendimiento; asegurar que la oferta esté articulada con el resto del sistema educacional y con el mundo del trabajo, propender a que exista permanente colaboración público privada (EL MERCURIO, 12 de Junio, 2016).

\section{Efectividad de la formación de enseñanza media técnico profesional}

Existe consenso mundial en considerar a la educación sistemática un factor clave para el desarrollo económico y social de los países. En este escenario, la formación profesional de enseñanza media adquiere una connotación muy relevante, más aún en los tiempos actuales en que las demandas al sistema económico guardan estrecha relación con la adecuada formación profesional y personal de quienes se desempeñan en las organizaciones laborales. Nos referimos al recurso o capital humano.

La enseñanza media técnico profesional (EMTP) tiene como misión formar a sus alumnos para que una vez egresados se incorporen al mundo del trabajo en condiciones tales que cumplan una función económicamente productiva y, a la vez, satisfagan sus necesidades. Además, el Estado de Chile ha establecido un segundo propósito alternativo para la EMTP, el que los egresados continúen estudios superiores, preferentemente en la especialidad en que se han formado en el establecimiento escolar.

En cuanto al logro de estas metas, la información con que se cuenta a nivel 
nacional confirma la existencia de dificultades. Ello tanto cuantitativa como cualitativamente. Respecto a lo primero, se registran importantes déficit en lo que concierne a la incorporación de los egresados al mundo del trabajo una vez que finalizan sus estudios secundarios, situación que se repite en lo que respecta a la continuación de estudios en la educación superior -centros de formación técnica, institutos profesionales, universidades-. En lo que se refiere al déficit de carácter cualitativo, la situación no es mejor. Ello se fundamenta, por una parte, en la opinión generalmente desfavorable de los empleadores en lo que concierne a la calidad de la preparación para el trabajo obtenida por los jóvenes, tanto respecto a las competencias duras -conocimientos- como a las blandas -valores y actitudes-. En lo que respecta a la continuación de estudios, las cifras estadísticas con que se cuenta y que se comentaban en el apartado anterior, no son positivas, tanto en cuanto a lo que se refiere al porcentaje de estudiantes proveniente de la EMTP como a su rendimiento, permanencia y titulación.

Aun cuando en los últimos años se han manifestado avances en ambas direcciones, la situación es todavía insatisfactoria, especialmente en lo que respecta a la ocupación de plazas de trabajo post secundaria y a la precariedad de éstas.

Luego, existe consenso en que en Chile se requiere aumentar significativamente la productividad laboral al mismo tiempo que otorgar efectivas oportunidades de trabajo adecuadamente remunerado a los titulados de los establecimientos técnico profesionales de enseñanza media. Ello, por diversos motivos, entre los que se destacan, por una parte los requerimientos del sistema económico de contar con recursos humanos eficientes y eficaces y, por otra, facilitar la movilidad social ascendente de un contingente cuyo perfil socioeconómico, en un $91 \%$ forma parte de los dos quintiles más pobres del país.

Ahora bien, desde la mirada de los potenciales empleadores, la actual formación de los egresados de la enseñanza media técnico profesional no es adecuada en términos de cubrir, como corresponde, los perfiles requeridos por el sector productivo y de servicios de recursos humanos técnicos de nivel medio. Esta situación influye negativamente en la economía del país, debido a que no se cuenta con suficientes trabajadores que formados en el sistema escolar posean un perfil, profesional y personal, que les permita enfrentar con éxito los desafíos cada vez mayores de una economía globalizada y, por tanto, altamente competitiva.

También esta situación afecta a las instituciones escolares TP y a su personal, dada la inseguridad de desarrollo y permanencia en el sistema que implica para estas organizaciones, con el consiguiente temor de inestabilidad en el empleo que puede provocar. Sin embargo, los mayores efectos negativos, sin duda, los asumen los alumnos, debido a que el obtener una formación profesional de calidad constituye su mejor oportunidad de movilidad socioeconómica.

Ahora bien, evaluaciones técnicas e investigaciones empíricas realizadas en Chile dan cuenta de que en lo que se refiere a la calidad de la formación profesional otorgada en los establecimientos escolares de enseñanza media, la situación no es adecuada, más bien y vista la efectividad de la misma, es insatisfactoria.

Información empírica que confirma lo dicho se presenta en una investigación realizada en la Región Metropolitana - la que concentra a más de la mitad de los establecimientos de formación técnica - . A continuación se transcribe parte de las conclusiones. 
- En consideración a los dos propósitos establecido para la enseñanza media técnico profesional, los cuales son formar a los alumnos para que una vez egresados del liceo se integren al mundo laboral o continúen estudios en la educación superior - universidad, instituto profesional, centro de formación técnica-, a la luz de la información obtenida en la investigación se puede concluir que al respecto la efectividad de la EMTP, en la Región Metropolitana, es relativamente baja, ya que menos de la mitad de los alumnos egresados en la promoción 2004, cuya situación pudo ser registrada, se encuentra trabajando o realizando estudios vinculados a la formación recibida en el liceo. De este segmento un tercio estudia, por lo que tiene un bajo logro el propósito de que los egresados continúen formándose en la educación superior. Un indicador que refuerza esta información es que en el año 2007 sólo el $28 \%$ de los egresados nacionales de la EMTP rindieron la prueba de suficiencia universitaria, (PSU), test obligatorio exigido para iniciar estudios universitarios. Por tanto, los resultados no son alentadores, debido a que la realidad no guarda relación con las expectativas de las autoridades del sistema escolar, de las autoridades de los centros escolares y de los propios alumnos y sus familias. (SERVAT, 2007, p. 43)

- Siendo altamente deseable para la sociedad en general y, en particular, para el sistema escolar, que los jóvenes que egresan de la enseñanza media continúen activos, es decir, estudien o trabajen, la gran cantidad de personas registradas en la investigación que se encuentran inactivas, no trabajan ni estudian, constituye una dificultad relevante, especialmente debido al perfil socioeconómico vulnerable de los egresados de los liceos de enseñanza técnico profesional. (SERVAT, 2007, p. 44)

- Habiéndose planteado en el estudio, la necesidad de dimensionar la efectividad de los sistemas de información administrativa de los liceos, en lo pertinente a la existencia y actualización de registros académicos y laborales de los egresados, se puede concluir que la situación al respecto es sumamente deficitaria, en casi todos los liceos en estudio, y sin diferenciación por modalidad de dependencia administrativa. (SERVAT, 2007, p. 45)

Un estudio realizado por el Ministerio de Educación, poco tiempo después, establece la existencia de problemas de calidad y pertinencia de esta modalidad educativa, en lo que respecta a la función que debiesen cumplir específicamente los liceos que imparten esta formación. Se agrega que no se cuenta en el sistema con mecanismos externos que permitan evaluar los resultados de aprendizaje, lo que dificulta la retroalimentación hacia los establecimientos para que pudiesen intervenir en la unidad escolar con estrategias de mejora de los déficits de gestión y docencia. Se señalan carencias pertinentes a la gestión de los establecimientos TP, principalmente vinculadas con el liderazgo efectuado por los equipos directivos y su capacidad para gestionar las relaciones del liceo con el entorno y el sector productivo de la localidad. También es relevante la crítica que se efectúa acerca de la gestión curricular y pedagógica que se realiza y la existencia de problemas en la capacidad de conducir tareas de evaluación, desarrollo y mejoramiento de los procesos y de los resultados en los establecimientos. (MINEDUC, 2008).

Posteriormente, un informe emanado del Ministerio de Educación señala lo siguiente:

En materia de aseguramiento de la calidad, se indica que la EMTP no cuenta con criterios definidos respecto a las condiciones mínimas que una unidad educativa debe cumplir para impartir una determinada oferta de especialidades y que sean verificadas durante su reconocimiento oficial. Asimismo, tampoco existe un mecanismo que permita monitorear el cumplimiento consistente de ciertos 
requisitos para la efectividad de los procesos formativos propios de esta educación. (MINEDUC, 2011, p. 4)

Son variados los factores que pueden originar la situación de significativos déficit en la formación TP, y las causas se encuentran en los diferentes espacios o niveles del Sistema Educacional, desde el estatal hasta el institucional (liceo), como también en las organizaciones laborales - productivas y de servicios - destino preferente de los egresados de la enseñanza profesional, y las instituciones de educación superior, especialmente centros de formación técnica e institutos profesionales.

Un aspecto muy relevante a evaluar en la formación técnico profesional de enseñanza media, es lo que concierne al grado de vinculación existente entre el liceo técnico y el laboral como también el nexo con la educación superior, todos ellos sectores e instituciones que constituyen el natural destino del alumnado TP.

En el estudio citado en líneas anteriores (SERVAT, 2007), se estableció que los establecimientos de enseñanza TP manifestaban muchas carencias en cuanto a mantención de contactos institucionales con organizaciones laborales concordantes con las especialidades que se imparten en la institución, y mínimos contactos con las instituciones de educación superior en la que potencialmente sus alumnos pudiesen continuar estudios. Ello, muy posiblemente, es una causa relevante de la insuficiente calidad de la formación profesional secundaria que se imparte en el país.

Dada la estructura de cargos existente en el sector productivo, parte importante de los trabajadores de la empresas debiesen provenir mediata o inmediatamente de la EMTP, sector de la educación que tiene como propósito preparar personal técnico de nivel medio, siendo su Misión socializar a sus alumnos en valores y actitudes e instruirlos en conocimientos y metodologías, que les posibiliten integrarse productivamente al mundo del trabajo una vez que egresen, mediata o inmediatamente.

En lo que respecta a la función que le atañe al sistema de educación superior, de cara a potenciar la calidad de la enseñanza técnico profesional de nivel medio, en los actuales tiempos ello se hace cada vez más relevante porque a diferencia de décadas pasadas, para sus egresados el continuar estudios se constituye en un propósito muy sentido, representando un destino deseable incorporarse a algún centro de formación técnica (CFT), instituto profesional (IP) o universidad. La precaria situación económica de la mayoría de los egresados TP representa un impedimento real para que este interés se materialice. Ello, aun cuando desde hace algunos años existe un sistema de becas y otros subsidios estatales que facilitan la incorporación y retención en el sistema a alumnos con carencias económicas. Sin embargo, esta ayuda, además de no ser suficiente, tampoco es extensiva a todos los interesados. De hecho, por el momento los estudiantes de centros de formación técnica y de institutos profesionales han quedado fuera del programa de gratuidad de la enseñanza superior que se ha iniciado en Chile el año 2016.

Se encuentra en los planes del Ministerio de Educación el ir incorporando, a partir del próximo año, 2017, a este importante beneficio a quienes realizan estudios de educación superior técnico profesional. Además del factor económico, las carencias existentes en la formación proporcionada en la mayor parte de los establecimientos de enseñanza media técnico profesional representan un real impedimento para sus egresados en cuanto no sólo a incorporarse, sino que también a mantenerse en el sistema y titularse.

Hist. Educ. (Online)

Porto Alegre

v. 21

ก. 52

Maio/ago., 2017

p. $111-135$ 
Considerando el destino de los egresados de los establecimientos de enseñanza media técnico profesional, referente a la continuación de estudios en la educación superior, sea en centros de formación técnica, institutos profesionales o universidades, desde hace ya algunos años ha aumentado significativamente el ingreso, llegando a cubrir alrededor del $40 \%$ de los egresados de liceos técnicos profesionales. Ello, sin duda constituye un avance importante. Sin embargo, según lo manifiestan autoridades educacionales, menos de la mitad de los que ingresan logran finalizar los estudios, obteniendo los respectivos títulos profesionales. Ello, constituye un problema tanto social como económico para los jóvenes afectados y sus familias, y para el país en su conjunto.

La percepción que sobre esta problemática se ha recabado desde actores profesionales del sistema escolar TP, refleja una situación aún peor. En un estudio se detectó que el $64.5 \%$ de los Directores de establecimientos técnico profesionales consultados y el $63 \%$ de los Jefes de Unidad Técnico Pedagógica de los mismos, manifestaron que a su juicio probablemente no más del $20 \%$ de sus egresados continuaría estudios superiores inmediatamente finalizada la educación secundaria. Es importante señalar que dicha percepción no correspondería a la de sus alumnos, ya que la mayoría de estos, en el mismo estudio, manifestaron expectativas positivas en términos de continuar estudios en la educación superior (SEPÚLVEDA; UGALDE; CAMPOS, 2008).

Ahora bien, el déficit en la formación técnico profesional sin duda obedece a diversas y complejas causas. Entre ellas, y muy principalmente, se encuentra la calidad de la gestión directiva de los liceos, incidiendo en ésta el tipo de liderazgo ejercido por las autoridades del establecimiento, la gestión del personal y la gestión técnico pedagógica. Además, y vinculada a la gestión, en este tipo de centro es muy relevante el tema de la existencia y eficacia de redes inter organizacionales. Como se señalaba en líneas anteriores, en el momento actual del país, constituye un hecho comprobado la falta de colaboración efectiva entre el mundo del trabajo y los establecimientos técnicos profesionales de enseñanza media.

Dicha colaboración debiese consistir, esencialmente, en un apoyo a la gestión directiva de los establecimientos, de cara a optimizar las decisiones, especialmente las pedagógicas -pertinentes a la formación y a la instrucción de los alumnos- mejorándose significativamente en calidad, oportunidad y pertinencia. En este nuevo escenario organizacional, sería posible esperar un avance significativo en la calidad de la formación que se imparte y, por ende, en su efectividad.

Partiendo del hecho comprobado empíricamente en estudios realizados en Chile, como lo señalábamos en páginas anteriores, en cuanto a que la colaboración entre el sector productivo y los establecimientos escolares TP, se encuentra ausente y/o carente de efectividad, a continuación se presenta información empírica referida a la temática, obtenida en una investigación Fondecyt realizada entre los años 2010 y 2013 (SERVAT; REYES; ZUÑIGA, 2013).

El estudio, de carácter nacional, versa acerca de la calidad del sistema de gestión directiva de los establecimientos de enseñanza media que imparten formación técnico profesional y el logro de sus objetivos.

A continuación se presentan, para su mejor comprensión, antecedentes metodológicos de la investigación. 
Las dimensiones objeto de estudio han sido: gestión del liderazgo, gestión del personal, gestión técnico pedagógica, logro de objetivos académicos especialmente referidos a la incorporación de los egresados al mundo del trabajo o a su continuación de estudios en la enseñanza superior.

La población considerada en el estudio se encuentra constituida por todos los liceos EMTP del país, excepto los de adultos: 907 establecimientos. La cantidad de liceos que conforman la muestra es de 83. El diseño muestral ha sido semi probabilístico, estratificado y multi etápico. La población fue estratificada por: modalidad administrativa municipalizada corporación, municipalizada DAEM, particular subvencionada, administración delegada -; sector geográfico - norte, centro, sur del país; zona - urbana, rural - y tamaño del establecimiento.

La información obtenida en la investigación, obedece a la medición, entre otras, de las siguientes dimensiones y variables:

- Respecto a la dimensión Gestión del Liderazgo, la variable:

- Adecuación de la conducción directiva de procesos con el entorno y comportamiento directivo innovador en la gestión de procesos con el entorno, con los indicadores: existencia de estrategias de vinculación institucional con organizaciones externas, existencia de convenios con otros establecimientos de enseñanza media técnico profesional, existencia de convenios con organizaciones empresariales y existencia de convenios con organizaciones de educación superior.

- Respecto a la dimensión Gestión Técnico Pedagógica, las variables:

- Calidad técnica de la instalación de los planes y programas de estudio, con los indicadores: retroalimentación desde las organizaciones empresariales afines, retroalimentación desde las organizaciones de educación superior afines, retroalimentación desde los egresados.

- Calidad de la planificación de la enseñanza, con el indicador participación de entidades externas en la planificación de la enseñanza.

- Calidad de los instrumentos de evaluación, con los indicadores: participación de entidades externas, y tipo de entidades en la elaboración de los instrumentos de evaluación.

- Existencia de programa adecuado de formación personal de los alumnos con los indicadores: retroalimentación del programa desde organizaciones empresariales, retroalimentación del programa desde organizaciones de educación superior.

- Calidad del programa de prácticas profesionales, con los indicadores participación de entidades externas en la planificación de las prácticas, participación de entidades externas en la evaluación de las prácticas y nivel de formalización institucional con organizaciones de prácticas.

Algunos de los resultados de la investigación, se transfieren a continuación: 
- La calidad de la gestión directiva es insatisfactoria, en general, en términos de la realización de los procedimientos pertinentes.

- En lo que respecta tanto a la calidad de la gestión directiva global, como a la calidad de la gestión de cada una de las dimensiones que la integran, se han manifestado déficits importantes.

- La dimensión de gestión relativamente mejor evaluada es la de liderazgo directivo, seguida de la gestión de recursos humanos y luego de la gestión técnico pedagógica, la cual aparece como la de menor calidad.

- En la dimensión gestión técnico pedagógica, existen diferencias notorias según sub dimensiones, siendo la peor evaluada la gestión curricular y la mejor la gestión de prácticas profesionales.

- Las variables "adecuación de la función directiva de procesos con el entorno" y "gestión innovadora" se presentan inadecuadas. Ello es muy preocupante debido al relevante efecto que tienen en la calidad de la formación profesional, especialmente en lo que guarda relación con su pertinencia y actualización.

(SERVAT et al., Informe Fondecyt № 1100473, 2013, sin numeración)

\section{Conclusiones}

Analizada la trayectoria de la enseñanza técnico profesional en Chile, en primer lugar es pertinente concluir que ésta ha sido relevante. Ello, se fundamenta en las características que ha asumido desde su origen hasta nuestros días. Es importante destacar el período histórico en que se inician las primeras actividades formales de carácter educativo tendientes a formar personal técnico, lo cual ocurre en el siglo XVIII, cuando Chile aún era una colonia del Reino de España. La trayectoria que desde entonces ha tenido la formación técnica en el país, ha sido desde el punto de vista cuantitativo, claramente ascendente, dada la tendencia creciente de creación de establecimientos escolares de esta modalidad desde el establecimiento de la República, e incluso antes de ello, hasta el presente con la existencia de un sistema de enseñanza técnico profesional en el que funcionan alrededor de un millar de establecimientos, a lo largo y ancho del territorio.

Cabe acotar que esta cifra es bastante relevante en un país como Chile que tiene una población pequeña, de alrededor de 17.000 .000 de habitantes. Actualmente, cerca de la mitad de la matrícula de los dos últimos cursos de la enseñanza media - 3o y 4ํ - está integrada por alumnos que estudian una especialización técnica.

Desde el punto de vista cualitativo o, en otras palabras, en términos de la efectividad de este tipo de enseñanza escolarizada, nos encontramos con diferencias de resultados a nivel nacional. Ello, tanto desde una perspectiva histórica o de la época en que se analice, como transversalmente $o$ atemporalmente.

Así es como, en los inicios de la formación técnica en el país, y especialmente durante el siglo IXX, funcionaron establecimientos educacionales emblemáticos, cuyo nivel de pertinencia y de calidad de la enseñanza que impartían se encuentra fuera de discusión. Tal es el caso de la importante función que desempeñó la Escuela de Artes y Oficios en el siglo XIX y XX, llegando a tener no solo reconocimiento en Chile sino que también a nivel internacional, lo que queda demostrado por el hecho de que parte de su alumnado provenía de otros países latino americanos. También es preciso destacar que dicho establecimiento influyó significativamente en la creación de la primera institución de educación superior de carácter técnico, la Universidad Técnica del Estado, la que llegó a tener gran prestigio nacional e internacional.

En lo que atañe a la situación de este tipo de enseñanza en las últimas décadas 
del siglo XX y hasta el momento actual, en términos de su efectividad, los estudios realizados avalan la percepción negativa que tienen diferentes tipos de actores relacionados con la formación técnico profesional de nivel secundario. Procurando identificar las principales causas de esta deteriorada imagen, postulamos que la gestión directiva de los establecimientos escolares TP en Chile, incide significativamente en los resultados académicos de los alumnos y con ello, en la efectividad de este subsistema escolar.

En páginas anteriores, hemos dado cuenta de evaluaciones realizadas por especialistas de la educación y resultados de estudios empíricos realizados en diferentes momentos durante este siglo, como también de las políticas nacionales establecidas por las autoridades educacionales del país, las que se refieren a procesos, planes y contenidos curriculares, metodologías y otros tópicos importantes referidas a este segmento educativo.

Ahora bien, a modo de conclusión, sostenemos que para lograr que los perfiles profesionales de los futuros técnicos de nivel medio correspondan a las expectativas de los actores comprometidos en su formación y a las exigencias de los potenciales empleadores y de las organizaciones de educación superior, se requiere urgentemente incorporar cambios relevantes.

En lo sustantivo, un foco relevante a intervenir abarca aspectos referidos a la gestión de los procesos que se realizan en el establecimiento educacional TP. Preferentemente la gestión del personal, del currículo, de la evaluación del rendimiento, de la orientación profesional y personal de los alumnos y de las prácticas profesionales.

Sin embargo, ello no es posible de realizarse sin que, simultáneamente, se incorporen cambios significativos en la gestión directiva global del establecimiento escolar, incorporando factores de innovación institucional, de participación pertinente de los distintos actores y de establecimiento de contactos fecundos con organizaciones externas. Respecto a éstas, especialmente aquellas con que naturalmente se debe vincular el establecimiento escolar dada la función que le corresponde cumplir. Es clave la existencia de contacto retro alimentador del liceo con el mundo laboral -principalmente con las empresas que funcionan en su región y comuna potenciando así el mejoramiento simultáneo de la calidad y de la efectividad de la educación - formación e instrucción del alumnado - y el logro de los objetivos de las organizaciones escolares. Es imperioso concebir al establecimiento educacional como una unidad abierta al medio externo, expectante acerca de los contactos interinstitucionales y de los beneficios que ello genera.

Luego, se requiere la búsqueda de instancias de colaboración entre la escuela y la empresa, que vaya más allá de la realización de las prácticas profesionales de los alumnos y de la donación de algunos bienes o dinero. De cara a lograrlo, es necesario generar espacios de encuentro en los que los actores provenientes de ambos sectores, elaboren y pongan en ejecución estrategias de colaboración orientadas a obtener mutuos beneficios, conjuntamente con el aporte a la sociedad que se produce en cuanto a impulsar el desarrollo económico del país. Dicha colaboración se debe circunscribir a los procesos y a los asuntos en los que la intervención de las organizaciones y los actores es posible y pertinente.

En cuanto a lo que se refiere al tipo de sujeto que, preferentemente, debe intervenir en la concreción de las alianzas entre el mundo educativo y el mundo laboral, 
por parte del liceo éstos debiesen ser preferentemente quienes ejecutan roles directivos y los encargados de las prácticas profesionales. Por parte de las empresas, los supervisores y los directivos de recursos humanos.

Ahora bien, para que exista un escenario de colaboración se requiere un cambio cultural, que involucre tanto a los actores del centro escolar como del sector laboral, lo cual no es fácil de efectuarlo ni es un proceso que dé frutos al corto plazo. Ello, porque implica modificación de valores, comportamientos y actitudes y cambio en las mutuas percepciones $\mathrm{y}$, a veces, desvalorizaciones recíprocas. Actualmente existe desconfianza y prejuicios negativos en actores del mundo educativo y del mundo empresarial. Suele suceder que los primeros tienden a considerar que se utiliza a los alumnos en práctica, los otros a que el trabajo que se realiza en los liceos no es de suficiente calidad.

De allí, que sea preciso plantear la relación en el marco de un contacto efectivo, en el que las percepciones e intereses particulares de cada sector den paso a un acuerdo en términos de facilitar el proceso de formación profesional y personal de los alumnos y, por ende, la efectividad de la formación técnico profesional.

En este escenario, es primordial tener presente la función y responsabilidad social que a les cabe unos y otros actores, en un mundo donde cada vez se hace más difícil el logro de los objetivos organizacionales e individuales y, a la vez, la sociedad exige mayores niveles de efectividad educativa y económica. De acuerdo a este escenario, las agencias involucradas - liceos y empresas -, debiesen en lo que corresponde, rediseñar sus planes, estructuras, roles y funciones.

Es también muy importante el aprovechamiento que se debe hacer, desde ambos sectores pero especialmente en los establecimientos escolares, de las redes de apoyo existentes, sean comunitarias, nacionales o de otra naturaleza. De no ser adecuadas en cantidad $y / o$ en calidad el Estado debiese financiar recursos humanos y financieros orientados a la elaboración y ejecución de proyectos de desarrollo tendientes a establecer estas redes a nivel comunal, provincial, regional y nacional.

Además, es urgente y de la mayor relevancia el establecimiento de políticas nacionales y la elaboración y aplicación de estrategias, que promuevan y faciliten una relación colaborativa efectiva entre directivos y docentes de liceos TP, por una parte y ejecutivos y supervisores de empresas, por la otra, de cara a elevar significativamente la calidad de la educación.

Situación similar debiese darse respecto a los necesarios vínculos entre el liceo técnico profesional y las instituciones de educación superior, especialmente los centros de formación técnica y los institutos profesionales, que a diferencia de las universidades constituyen el destino natural de continuación de estudios de los egresados.

Luego, la participación pertinente y eficaz de los actores institucionales en los procesos organizacionales, como la colaboración de los agentes externos en la gestión administrativa y pedagógica del liceo técnico profesional, no representa una opción, sino, un camino necesario para subsistir y evolucionar en un mundo muy diferente al existente en siglos pasados, en un escenario económico y laboral cada vez más competitivo, con muchas turbulencias generadas dentro de las instituciones, en la sociedad y más allá de sus fronteras. 


\section{Bibliografía}

BIBLIOTECA NACIONAL DE CHILE. Corporación de Fomento de la Producción (19391952). 2016. Recuperado de: <http://www.memoriachilena.cl/602/w3-article-html>.

De la Escuela de Artes y Oficios a la Universidad de Santiago. La Educación Técnica en Chile. 2016. Recuperado de: <http://www.memoriachilena.cl/602/w3-article 682.html>.

CALISTO, R.; CID, J.; CUADRA, L.; CUEVAS, S. Desafíos y perspectivas de la enseñanza media técnico profesional en la Región del Bío-Bío: Investigación sobre las necesidades de perfeccionamiento docente. Universidad de Concepción: Concepción, 2014.

COLEGIO DE PROFESORES DE CHILE (2007). Sin título, citado en Calisto et al., 2014.

EL MERCURIO. Mineduc diseña institucionalidad para fortalecer la educación técnica. Santiago de Chile, 12 de junio de 2016.

LÓPEZ, E. Sin fecha, citado en Calisto et al. Concepción, 2014.

MINISTERIO DE EDUCACIÓN. (Sin fecha), citado en Calisto et al.. Concepción, 2014.

Bases Curriculares de la Formación Diferenciada Técnico Profesional.

Especialidades y Perfiles de Egreso. Santiago de Chile, 2013.

Nuevas Bases Curriculares TP. 2013. Recuperado de: <http//www.

mineduc.2013. nuevas bases curriculares.cl>

Centro de Estudios. Educación Técnica Profesional en Chile. Antecedentes y claves de diagnóstico. Santiago de Chile, 2011.

Chile, 2008.

Bases para una política de formación técnico-profesional en Chile. Santiago de

Evaluación del Marco Institucional de la Educación Media Técnico-Profesional. Santiago de Chile, 1998.

. La Educación Media para el trabajo. Santiago de Chile, 1992.

MUÑOZ, J. et al. La Universidad de Santiago de Chile. Editorial USACh: Santiago de Chile, 1987.

SCHIRO, M. Teoría Curricular. Visiones en conflicto y preocupaciones permanentes. Boston College: Sage Publications, California, 2008.

SEPÚLVEDA, L.; UGALDE, P.; CAMPOS, F. Estado y perspectivas de la enseñanza media técnico profesional en Chile: Un estudio sobre las orientaciones estratégicas predominantes en los actores. Fonide, CIDE, Universidad Alberto Hurtado: Santiago de Chile, 2008.

SERVAT, B. Efectividad de la enseñanza media técnico profesional. Informe Final de Investigación. Pontificia Universidad Católica de Chile, Facultad de Educación. Santiago de Chile, 2007.

SERVAT, B.; REYES, J.; ZUÑIGA, M. Gestión directiva de liceos de enseñanza media técnico profesional y logro de objetivos académicos. Diagnóstico y propuestas de sistemas de estrategias de gestión, innovadoras e inclusivas. Informe Final Proyecto Fondecyt № 1100473. Santiago de Chile, 2013.

SUPREMO GOBIERNO DE CHILE. Crónica de la Escuela de Artes y Oficios para Mujeres. En Boletín de la Sociedad de Fomento Fabril. Tomo V. Santiago de Chile. 1888. Recuperado de <http://www.memoriachilena.cl/602/w3-article-75672 html>. 
Reglamento para la Escuela de Artes y Oficios. Imprenta Nacional. Santiago de Chile. 1864. Recuperado de <http://www.memoriachilena.cl/602/w3-article-95225.html>.

BERTA SERVAT POBLETE es Socióloga, Licenciada en Sociología y Magister en Ciencias de la Educación de la Pontificia Universidad Católica de Chile, Especialista en Recursos Humanos de la Universidad de Chile, Doctora en Ciencias de la Información y Comunicación de Masas de la Universidad Complutense de Madrid. Su trayectoria académica comprende la docencia, investigación y publicación, como Profesora de Planta en la Pontificia Universidad Católica de Chile y como Profesora Invitada en otras universidades chilenas y de otros países.

Dirección: Carlos Silva Vildósola, 7897, Santiago de Chile, Chile.

E-mail: bservat@puc.cl

Recebido em 18 de agosto de 2016.

Aceito em 13 de outubro de 2016. 